\title{
Percepción del emprendimiento caficultor en la región Huasteca, centro de México
}

\author{
Cruz García Lirios ${ }^{1}$ \\ Javier Carreón Guillén ${ }^{2}$ \\ José Marcos Bustos Aguayo \\ Jorge Hernández Valdés ${ }^{4}$
}

Recibido: 15 de noviembre de 2019

Aprobado: 13 de diciembre de 2019

García Lirios, C., Carreón Guillén, J., Bustos Aguayo, J. M. y Hernández Valdés, J. (2020). Percepción del emprendimiento caficultor en la región Huasteca, centro de México. Revista Activos, 18(1), 235-266. DoI: https:// doi.org/10.15332/25005278/6164

\section{Clasificación JEL: L26, O13}

1 Estudios de Doctorado en Psicología Social y Ambiental, Universidad Nacional Autónoma de México. Profesor de asignatura, Universidad Autónoma del Estado de México-Unidad Académica Profesional Huehuetoca. teléfono: 56226666 extensión 47 385. Correo electrónico: garcialirios@yahoo.com ORCID: http://orcid.org/0000-0002-9364-6796

2 Doctor en Administración y profesor titular "C", Universidad Nacional Autónoma de México, Escuela Nacional de Trabajo Social. Adscrito al Sistema Nacional de Investigadores. Correo electrónico: javierg@unam.mx

3 Doctor en Psicología y profesor titular "C", Universidad Nacional Autónoma de México, Facultad de Estudios Superiores Zaragoza. Correo electrónico: marcos.bustos@unam.mx

4 Maestro en Educación y profesor titular “A”, Universidad Nacional Autónoma de México, Escuela Nacional de Trabajo Social. Correo electrónico: jorheval@unam.mx 


\title{
Resumen
}

El objetivo del presente trabajo fue especificar un modelo para el estudio del emprendimiento caficultor. Se realizó una investigación transversal y exploratoria con una selección no probabilística de 300 comerciantes del café. A partir de un modelo estructural se estableció la confiabilidad y validez de cinco dimensiones alusivas a percepciones de oportunidad económica, financiera, de ventas, social y ambiental. El cuarto factor reflejó el constructo, seguido del primero y el quinto. Con base en los marcos teóricos y conceptuales, se propone el estudio de la percepción emprendedora en grupos vulnerables que se dedican a la venta del café como un estilo de subsistencia. Los hallazgos permiten establecer un vínculo entre la teoría de la elección racional y la teoría del capital humano con respecto a la teoría de los bienes comunes y la teoría del emprendimiento social como marcos conceptuales para resaltar la intervención del trabajo social.

Palabras clave: emprendimiento social, financiamiento, venta, ambiental.

\section{Perception of Coffee Entrepreneurship in the Huasteca Region, Central Mexico}

\begin{abstract}
The aim of this paper is to specify a model for the study of coffee entrepreneurship. A cross-sectional and exploratory research was conducted with a non-probabilistic selection of 300 coffee merchants. A structural model established the reliability and validity of five dimensions referring to perceptions of economic, financial, sales, social, and environmental opportunities. The fourth factor reflected the construct, followed by the first and fifth factors. Based on theoretical and conceptual frameworks, this paper proposes to study entrepreneurial perception in vulnerable groups engaged in the sale of coffee as a means of subsistence. The findings make
\end{abstract}


it possible to establish a link between the theory of rational choice and the theory of human capital with respect to the theory of common goods and the theory of social entrepreneurship as conceptual frameworks to highlight the intervention of social work.

Keywords: social entrepreneurship, financing, sale, environmental.

\section{Introducción}

Hasta el momento en que se redacta este trabajo, un tercio de la población mundial ha sido confinada como política de mitigación pandémica ocasionada por el coronavirus SARS-COV-2 y la covid-19, que han infectado a ocho millones de personas, enfermado a cuatro millones y han cobrado la vida de medio millón. Ante este panorama, el emprendimiento parece ser una respuesta social ante ambas crisis la sanitaria y la económica.

La crisis económica ha propiciado la caída de las economías emergentes y regionales en las que destaca México y la región turística de la Huasteca Potosina. En virtud de que el $60 \%$ de la economía nacional es de carácter informal, la crisis económica se exacerbó con la pérdida del 75 \% del turismo a nivel nacional y alrededor de $3.5 \%$ a nivel regional.

Por consiguiente, el emprendimiento social ha sido identificado como una estrategia de desarrollo local ante la crisis sanitaria y la crisis económica que se desenvuelve en la región a partir de marzo cuando los indicadores evidenciaron un decrecimiento de la actividad turística por motivos relacionados con la pandemia, el confinamiento de las personas y la recesión de la economía.

El objetivo del presente trabajo fue especificar un modelo para el estudio del emprendimiento de la caficultura y los servicios asociados como derivados. A partir de un marco teórico, conceptual y empírico se proponen los ejes y temas de una agenda de desarrollo local. En seguida, se aborda 
la problemática desde un método de cuantificación e instrumentación de un cuestionario. Posteriormente, se exponen los resultados con estadísticas descriptivas e inferenciales y, finalmente, se discuten los hallazgos con el desarrollo endógeno a fin de poder delinear la planificación estratégica de un programa de emprendimiento.

El aporte del presente trabajo consiste en: (a) revisión documental de la literatura sobre emprendimiento local; (b) especificación teórica, conceptual y empírica de un modelo para el estudio del fenómeno; (c) abordaje metodológico para la explicación del emprendimiento caficultor; (d) diagnóstico de la dimensión perceptual del emprendimiento caficultor; (e) discusión de los resultados con base en los ejes y temas de agenda local, y (f) recomendaciones de aplicación e implementación del emprendimiento caficultor a la región

\section{Revisión documental}

\section{Sociohistoria del trabajo social y emprendimiento en México}

En México, la sociohistoria del trabajo social está vinculada con algún tipo de emprendimiento si se considera que este consiste en iniciativas emanadas de la sociedad civil con respecto a sectores excluidos del desarrollo (García, 2020).

Posterior a la Revolución mexicana de 1910, los gobernantes tomaron decisiones que orientaron su relación con los gobernados. En este sentido, la Expropiación Petrolera de 1936 es un claro ejemplo de la política social que la clase política implementó en los sectores civiles al organizarlos en grupos. En este escenario, el emprendimiento social fue activado por una política expropiadora ya que se realizó una colecta nacional para subsanar las indemnizaciones a las compañías de petróleo que controlaban la extracción y comercialización del hidrocarburo. 
De este modo, el trabajo social que emergió con la gestión y administración de los gobiernos posteriores a la guerra civil se caracterizó por (a) adscripción a una ideología revolucionaria y caudillista como sustento de una identidad nacional; (b) inclusión de sectores burócratas, obreros, campesinos y empresariales, pero exclusión de sectores pobres, y (c) alineación con un presidencialismo metaconstitucional (García, 2014). En ese proyecto de nacionalismo expropiador, sectores sociales fueron confinados a un emprendimiento local semiarticulado con financiamiento proveniente de remesas. Las comunidades administraron estos fondos mediante redes cooperativas, pero la cooptación del Estado en clientelas políticas redujo esta alternativa a su mínima expresión.

Es decir que el trabajo social fue un instrumento de gestión e interlocución con los sectores que firmaron la carta magna posterior a la guerra civil (Bustos, 2020). Por consiguiente, los profesionistas del trabajo social se alejaron de toda iniciativa civil al socavar los lineamientos institucionales del Estado posrevolucionario. El capital de remesas se siguió orientando al emprendimiento comunitario, pero limitado por los usos y costumbres de las cooperativas locales.

Durante la fase sociohistórica de prosperidad económica, laboral y educativa del periodo 1940 a 1960, mejor conocida como Milagro Mexicano, el trabajo social alcanzó su mayor esplendor bajo las políticas de salud que permitieron la creación de instituciones emblemáticas, tales como el Instituto Mexicano del Seguro Social (IMSs), en 1943, y el Instituto de Seguridad de Servicios Sociales de los Trabajadores del Estado (ISsSTE) en 1959.

Sin embargo, los profesionistas del trabajo social fueron confinados a programas de combate a la explosión demográfica o estrategias de salud reproductiva y sexual, así como a prevención de enfermedades y accidentes en la naciente industrialización y sus efectos sobre la salud ocupacional de los trabajadores del Estado (García, 2015). El emprendimiento local quedó acéfalo y la desarticulación entre el sector público con las micro-, pequeñas y medianas empresas avanzó hacía una relación asimétrica entre actores políticos y sociales. 
Es posible observar que, durante el periodo en estudio, el emprendimiento social se gestó en las comunidades y localidades, ya que se redujo a su mínima expresión en las urbes (Carreón et ál., 2014). Significa entonces que el desarrollo comunitario se concentró en las actividades agroindustriales y el turismo como un efecto de las políticas sociales de fomento empresarial. En este marco, el trabajo social estableció los lineamientos para la clasificación de los sectores excluidos, marginados y vulnerados en sus derechos económicos.

Fue a partir de conflictos entre el estado y la sociedad civil que el emprendimiento social emergió como una respuesta no concertada por políticas públicas en las que el trabajo social optó por reproducir la ideología del Estado (Juárez, 2020). Se trató de un proceso de propaganda del nacionalismo caudillista que concentró a los sectores en confederaciones con el fin de poder controlar sus cadenas de financiamiento y producción agroindustrial, o bien, en el caso del turismo establecer las denominaciones de pueblos mágicos para activar la economía regional.

De este modo, los movimientos sociales antecedieron al emprendimiento social en virtud de que suponen estilos de organización que explican la conformación de esferas y redes civiles (Sandoval, 2020). El capital semilla para los proyectos cooperativos provino de las remesas y se destinó a las empresas familiares sin conexión alguna con las multinacionales salvo en el caso del café como un valor agregado del turismo.

Es el caso del conflicto entre estudiantes y Gobierno, en 1968, a partir del cual las estructuras sociales optaron por un cambio paulatino que se consolidó en 1985 con la movilización civil ante los desastres del sismo más mortal que registra la ciudad de México (García et ál., 2014). Es decir, la confianza en el Gobierno se redujo a su mínima expresión, a la vez que la organización sectorial emergió como alternativa a la gestión pública. La nueva autogestión social se desarrolló y consolidó como un proyecto de emprendimiento social para el desarrollo de barrios y comunidades aledañas a las urbes, o bien al interior de las ciudades como espacios multiculturales e interétnicos. 
No obstante que la carta magna es innovadora en cuestiones de propiedad y administración de ejidal, la movilización campesina se orientó por su disidencia a las políticas públicas, ya que las oportunidades de desarrollo se limitaron a las plantaciones extranjeras (Coronado, 2019).

La producción y comercialización del sector agroindustrial se gestó en redes para transferir el conocimiento técnico a nodos centrales de emprendimiento. Las cooperativas exitosas muy pronto socavaron sus relaciones con los micro-, pequeñas y medianas empresas en un proyecto de crecimiento interno.

Es así como el emprendimiento surge en las urbes como una respuesta al autoritarismo del Estado más que como un síntoma de prosperidad, calidad de vida y bienestar, aun cuando el país ha tenido relaciones comerciales con Estados Unidos. Incluso en el periodo que inicia el tratado de libre comercio, el emprendimiento parece ser exclusivo de trasnacionales más que de empresas locales o cooperativas -7 de cada 10 nuevas empresas desaparecen en los dos primeros años luego de haber esperado el mismo tiempo para alta en la hacienda pública一.

En este breve recorrido sociohistórico, el trabajo social adquirió un sentido de bienestar debido a la política sustitución de importaciones, pero se desvinculó de los sectores desfavorecidos, aunque el emprendimiento de la sociedad civil provino de diversos sectores, la relevancia histórica del trabajo social emerge de las movilizaciones civiles urbanas o semiperiféricas (García et ál., 2015).

Por consiguiente, el estudio del emprendimiento en comunidades es un tema emergente y lo es más aún con la intervención del trabajo social; empero, durante el periodo expuesto, los gobiernos del centro de México han desarrollado esquemas de apoyo a la agroindustria, principalmente en lo relativo al café. 


\section{Emprendimiento en México}

El censo económico de México en el 2009 fue llevado a cabo con el 99,9 \% (3 587 979) de las empresas que son de tipo uniestablecimiento y el $1 \%(39080)$ es multiestablecimiento. $17.9 \%$ (6979) son nacionales, $15 \%$ (5488) son locales y $67.1 \%$ (26246) son multinacionales (Inegi, 2010).

Es decir que los indicadores económicos muestran que la sistematización del emprendimiento se gesta en las trasnacionales, pero se lleva a la práctica en las micro-, pequeñas y medianas empresas (mipymes). Esto es así porque el desarrollo económico puede estar interrelacionado por principios de utilidad y ganancia, pero ambos pueden ser transformados en discursos, estilos de vida y consumo específicos de comunidades y localidades ante una crisis de abastecimiento de recursos naturales y servicios públicos correspondientes. Ante tal escenario emerge el emprendimiento como una respuesta de la sociedad civil ante la gestión y administración poco atingente del Estado (García, 2014).

Existen 4145772 micro-, pequeñas y medianas empresas que ocupan a 19179350 empleados, de los cuales 5073432 laboran en 489532 empresas del sector maquilador e industrial, 6389648 se ocupan en 2042 641 empresas de comercio y 7716270 laboran en 1613601 empresas no financieras del sector privado. En otras palabras, el $21.6 \%$ de las empresas creadas en 2013 ocupa menos de un empleado.

En otras palabras, es posible observar una tendencia de emprendimiento social que, no obstante, su baja productividad, genera redes sociales de cooperación solidaria. Es decir, que, ante una crisis económica o política, algunos sectores emprendedores de la sociedad civil renuncian a la lógica de la ganancia y la utilidad para redistribuir los costos y beneficios en un mayor porcentaje de la población (García et ál., 2015).

Las microempresas representan el $95.9 \%$ (3 976 912) del total y ocupan al $43.7 \%$ (12 899 155) de la fuerza laboral. La pequeña empresa representa el $3 \%$ (126 262) y ocupa al $13 \%$ (2 496835 ) de la fuerza laboral y la mediana 
empresa representa el 0,7\% (27 706) y ocupa el $10.6 \%$ (2 023676 ) de la fuerza laboral (Inegi, 2010).

En el caso de San Luis Potosí (centro de México), el emprendimiento social está centrado en las oportunidades y las capacidades de las microempresas que, por su lógica de colaboración solidaria, tienden a expandirse al reducir sus utilidades y ganancias (García, 2015).

En el sector de manufactura las microempresas representan el $93.6 \%$ (458 096) y son el $20.8 \%$ (1 057 456) de la fuerza laboral, las pequeñas empresas representan el $4.2 \%$ (20 455) y ocupan al $8.8 \%$ (446 181) de la fuerza laboral y las medianas empresas representan el 1,5 \% (7 441) y ocupan al 16,8 \% (851 506) de la fuerza laboral (Inegi, 2010).

En el sector comercio las microempresas representan el $96.9 \%$ (1978 887) y ocupan al 60.5\% (3866 223) de la fuerza laboral, las pequeñas empresas $2.2 \%$ (43 967) y ocupan al $11.7 \%$ (745 253) de la fuerza laboral y las medianas empresas representan el $0.7 \%$ (14 454) y ocupan al $12 \%$ (764 763) de la fuerza laboral (Inegi, 2010).

A diferencia del sector maquilador, el comercio supone una mayor participación de emprendedores, ya que las redes de producción de las que depende la prosperidad de maquiladores, no es un requerimiento en la compra y venta de productos al mayoreo o menudeo. Es decir, que el emprendimiento local está orientado por la cultura laboral de las microempresas, muchas de ellas extraídas de las comunidades dedicadas a la comercialización del café y sus derivados (Carreón et ál., 2014).

En San Luis Potosí, estado del centro de México, se ubica el 2.1 \% de las mipymes de las cuales el $1.8 \%$ está en el sector maquilador y el $2 \%$ está en el sector comercio y $2.2 \%$ en el sector no financiero privado. El sector comercio representa el $47.2 \%$ (41 640) y ocupa al $29.7 \%$ (124 897), el sector servicios representa el $40.9 \%$ (36 066) y ocupa al $32.2 \%$ (135 353) y el sector manufacturero el $10 \%$ (8852) y ocupa al $29.7 \%$ (125 011) de la fuerza laboral (Inegi, 2010). 
En efecto, a partir de la dinámica económica de mipymes en San Luis Potosí, es posible advertir que se ha estado gestando un emprendimiento en función del crecimiento de servicios, pero a nivel de microempresas. Es decir que la caficultura tiene mayores oportunidades de comercialización en un escenario donde la actividad comercial supera en dimensión y fuerza laboral a la industrial (García et ál., 2014).

En el sector comercio, las microempresas representan el $96.8 \%$ (40319) y ocupan al $61.9 \%$ (77 284) de la fuerza laboral, las pequeñas empresas representan el $2.2 \%$ (928) y ocupan al $12.7 \%$ (15 907) de la fuerza laboral y las medianas empresas representan el $0.7 \%$ (298) y ocupan al 12,8 \% (15 949) de la fuerza laboral local (Inegi, 2010).

Si el mercado local en cuanto a la comercialización de productos y servicios vinculados con el café está condicionado por habilidades sociales tales como el establecimiento de redes de comercio, entonces las localidades y comunidades son un escenario idóneo para establecer algunas diferencias en cuanto a la percepción de oportunidades de venta del café y sus derivados (García et ál., 2015).

En cuanto a la demanda laboral, las mipymes advierten que el dominio de un segundo idioma es la habilidad más requerida $(M=3.6)$, seguida de la comunicación escrita $(M=2.64)$ y la facilidad para relacionarse $(M=2.31)$.

Las actitudes requeridas por las mipymes son visión internacional $(M=2.99)$, aprecio por la cultura $(M=2.98)$ y respeto al medio ambiente $(M=2.38)$.

\section{Emprendimiento en San Luis Potosí}

El estado de San Luis Potosí colinda con los estados de Zacatecas, Nuevo león, Tamaulipas, Guanajuato, Querétaro, Hidalgo y Veracruz.

El estado cuenta con 58 municipios, sus coordenadas lo ubican 24 grados y 29 minutos al norte; al sur, 21 grados y 10 minutos latitud 
norte; al este 98 grados y 20 minutos, y al oeste 102 grados y 18 minutos longitud oeste.

El Cerro Grande es su mayor elevación con 3180 m s. n. m., seguido de la Catorce $3101 \mathrm{~m}$ s. n. m. y la Cierra Coronado m s. n. m. msnm. Predomina un clima seco templado con el $27.7 \%$, seguido de un clima seco semicálido, con el $20.1 \%$, y semiseco templado, con el $12.8 \%$. La temperatura promedio es de 25.3 grados Celsius y su precipitación media anual es de $978.8 \mathrm{~mm}$ en una superficie territorial de $60982.8 \mathrm{~km}^{2}$ (el $3.1 \%$ del territorio nacional).

El estado de San Luis Potosí ocupa el lugar 19 con una población total de 2.586 millones de habitantes, y tiene una tasa de crecimiento del $1.4 \%$ y una densidad de 4.2 habitantes por kilómetro cuadrado.

Real de Catorce cuenta con una población total de 9716 habitantes de los cuales 4932 son hombres y 4784 son mujeres. En Xilitla habitan 51 498, de los cuales 25484 son hombres y 26014 son mujeres; 30,4 \% de la población es menor de 15 años. $10.7 \%$ de la población emigró al estado; 3,7 \% de sus habitantes no residía en estado y 0,1 \% del total de la población es migrante. Asimismo,10.7 \% de la población habla una lengua local, 137682 hablan el nahualt, 96568 hablan el huasteco y 10807 el pame.

La tasa de natalidad es del 20.1 por cada mil habitantes, las mujeres tienen el 2.1 en promedio de hijos nacidos vivos y la densidad poblacional es de cuatro personas por vivienda.

El $7.9 \%$ de la población total es analfabeta, el $90.6 \%$ de la población entre 5 y 14 años asiste a la escuela y las mujeres representan el $40 \%$ de la fuerza laboral.

\section{Trabajo social en el desarrollo local}

Las teorías que explican la intervención del trabajo social para el desarrollo local en general y comunitario en particular son: la teoría de la elección 
racional, la teoría del capital humano, la teoría de los bienes comunes y la teoría del emprendimiento social.

Grosso modo, la teoría de la elección racional plantea que el desarrollo de una comunidad se gesta desde la selección de oportunidades y capacidades, ambas orientadas al logro de objetivos y metas a corto, mediano y largo plazo (García et ál., 2014). Desde la óptica de la elección racional, el emprendimiento social es asumido como resultado de las oportunidades brindadas por las políticas de fomento empresarial, o bien por las políticas de reactivación económica.

En el primer caso, el emprendimiento es de corte socioestatal, ya que es el gobierno local o federal el que impulsa la denominación de pueblo mágico para reactivar la economía a partir del turismo nacional e internacional. En el segundo caso, luego de la crisis sanitaria, financiamiento a empresas comunitarias y cooperativas familiares es la divisa principal de programas y estrategias para la reactivación de la economía.

En ese sentido, existe una diferencia sustancial entre racionalidad e irracionalidad. Es el caso de la toma de decisiones unilaterales o mayoritarias que por su grado de exclusión de minorías - comunidades migrantes o locales - que tienen valores diferentes a la utilidad y la ganancia, así como visiones distintas de propiedad privada (García et ál., 2015). Las cooperativas familiares y las empresas comunitarias son producto de elecciones de oportunidades cuando el capital semilla proveniente de las remesas se asocia a la mano de obra familiar y la fuerza laboral endógena. En ambos casos, el emprendimiento social es activado por actores mediadores de las necesidades y expectativas locales con respecto a los fondos disponibles de familias administradoras de remesas.

Por consiguiente, la teoría del capital humano, en términos generales, intenta reducir las diferencias entre gobernantes y gobernados, mayorías y minorías, oportunidades y capacidades a partir de un sistema de gestión y administración de bienes públicos. Es decir, al asumir como propiedad del Estado los recursos, el gobierno federal o local está obligado a distribuir 
equitativamente los costos y beneficios relativos al desarrollo (Carreón et ál., 2014). Las empresas familiares ejemplifican este proceso de cogestión en el que las autoridades locales organizan a los productores en tandas de financiamiento. En cambio, las empresas comunitarias interiorizan sus recursos a partir de uso y costumbres más que desde la administración semipública.

Sin embargo, la teoría del capital humano considera que quienes no están dispuestos a contribuir, aunque reciban beneficios, son parte del desarrollo (Molina, 2020). Es el caso de la fuerza laboral y la mano de obra proveniente de municipios periféricos a las plantaciones agroindustriales. En el caso de la caficultura, la concentración del financiamiento público en los pueblos mágicos y los fondos provenientes de remesas familiares hace posible que se estructure una centralidad productiva y comercializadora que se alimenta de migrantes aledaños.

Por lo tanto, la teoría de la elección racional y la teoría del capital humano advierten que el problema del desarrollo local a partir de la gestión y administración de los bienes y recursos considerados públicos estriba en las decisiones irracionales y la no cooperación (Adams, 2020). Esto es así porque el emprendimiento social requiere del concurso de sectores públicos y privados, actores políticos y sociales en torno a una gobernanza de los recursos comunes. De esta manera, las partes interesadas acuerdan responsabilidades compartidas en objetivos, tareas y metas.

Es la teoría de los bienes comunes la que resuelve el dilema del desarrollo local a partir de una racionalidad que supone el abandono de usos y costumbres asumidas como irracionales — gestión y administración de dinero para festividades en lugar del servicio de agua potable - o bien el dilema que consiste en cooperar, aun cuando algunos no contribuyan y se beneficien del sistema (García, 2014).

La teoría de los bienes comunes advierte que el desarrollo parte del tipo de bienes: 1) privados, donde la seguridad de la propiedad o recursos están garantizados por las leyes que el Estado debe hacer cumplir mediante 
programas, estrategias e instrumentos, pero excluye a sectores civiles que no tienen ese tipo de bienes; 2) públicos, donde el Estado garantiza la distribución de los costos y beneficios, pero es susceptible de corromperse, y 3 ) comunes. los recursos suponen oportunidades en función de las capacidades de organización y cooperación social.

De este modo, el desarrollo local que está determinado por la lógica de los bienes comunes requiere de una gestión en comunidades mediante la promoción de la solidaridad y la cooperación (García, 2015). En este contexto, el emprendimiento social encarna ambas virtudes comunitarias al prevalecer la confianza social, la empatía de las partes interesadas y el compromiso de los actores implicados.

Se trata de un círculo virtuoso de oportunidades e innovaciones desde y para la comunidad, aunque las familias que proveen los créditos derivados de las remesas se enlazan con las cooperativas a través de los usos y costumbres locales.

Precisamente, la complejidad de esta fase sociohistórica en la que una comunidad con valores biosféricos debe asumir una dinámica productiva antropocéntrica supone una promoción de relaciones de emprendimiento social (Anguiano, 2020). El biosferismo no solo es parte de las tradiciones y normas de la comunidad, aglutinando los objetivos, tareas y metas a partir de recursos y futuro comunes. El rasgo distintivo del emprendimiento comunitario radica en este biosferismo como representación de un propósito común.

En el caso de la caficultura, el emprendimiento social va más allá de una superación de capacidades con respecto a oportunidades, pues supone el establecimiento de una agenda común entre sectores civiles, políticos y económicos orientados a la gestión de un conocimiento suficiente para el desarrollo responsable. 


\section{Marco teórico}

\section{Teoría del emprendimiento social}

En contextos de salud comunitaria afectada por inundaciones, incendios o heladas, la comercialización del café resulta ser un ejemplo que seguir para observar el emprendimiento local. En torno al emprendimiento del café, los datos parecen confirmar los supuestos de a teoría del emprendimiento (Vázquez et ál., 2016). Las oportunidades económicas — subsidios de los gobiernos federal y local para incentivar la agroindustria y comercialización-y las oportunidades financieras - créditos a partir de balances y prospectivas de la comercialización de productos y servicios- determinan las percepciones de oportunidad de ventas al menudeo - expectativas de mayores ganancias ante costos mínimos- (Acar y Acar, 2014).

Sin embargo, algunos estudios destacan la importancia de las redes sociales de emprendimiento como factores potenciadores de la venta solidaria de productos y servicios locales ante el arribo de trasnacionales y sus posibles afectaciones a la economía municipal (Cruz et ál., 2016).

La teoría de las redes de emprendimiento advierte que la cooperación y la solidaridad se gestan en escenarios de vulnerabilidad económica y financiera (Robles et ál., 2016). Es decir que, ante el embate de las trasnacionales, los comerciantes se organizan para hacer un frente común en la defensa del mercado local (Hernández y Valencia, 2016). De este modo, los liderazgos y seguidores estructuran redes de información en torno a los precios de venta, promociones y valores agregados en función de la competencia trasnacional (Omotayo y Adenike, 2013).

En tal sentido, las redes de emprendimiento resaltan la importancia de la cooperación y la solidaridad, y vinculan sus productos y servicios a otras actividades tradicionales de la localidad (Escobar, 2014). Las cooperativas en torno a la producción y comercialización del café y sus derivados se relacionan con las asociaciones de hoteles, restaurantes y taxis para 
ofrecer como valor agregado al servicio turístico los productos del café en sus diferentes presentaciones. Se trata de un emprendimiento orientado hacia la relación biosférica con el entorno en el que el café es un símbolo distintivo de la región.

Un nuevo tipo de emprendimiento, derivado de las redes sociales es el ambiental (Mendoza et ál., 2016). Se trata de expectativas de comercio de productos y servicios en función del conocimiento del clima local, los espacios, usos y costumbres de la comunidad (Saansongu y Ngutor, 2012). Además, se edifica un círculo virtuoso de símbolos, significados y sentidos de relaciones comunes con el entorno. Es decir, la caficultura es parte del turismo y este del emprendimiento de productos derivados.

La teoría del emprendimiento ambiental explica la asociación entre comerciantes, la diversificación de sus productos y servicios, así como el consumo de estos en contextos específicos y condiciones ambientales extremas (Anicijevic, 2013). Se trata de decisiones en situaciones de altos riesgos de las que se esperan mayores ganancias. Es el caso de los productos derivados del café que suponen un riesgo de inversión cuantioso por la adquisición de procesadoras o empacadoras, pero también general cuantiosas ganancias en la venta de café orgánico.

De este modo, el café resalta como un producto y servicio adaptable a los efectos del cambio climático en la salud comunitaria donde estar activo es un requerimiento para subsistir ante sequías, inundaciones, deslaves, heladas o incendios (Quintero et ál., 2016). Además, quienes deciden comercializar los productos derivados del café compiten con otros productos en el mercado local, regional e internacional. La cerveza y las bebidas azucaradas son ampliamente consumidos y restan ventas al café. Por consiguiente, el emprendimiento de este producto se realiza a partir de sus ventajas competitivas como la diversificación de su contenido o la innovación de otros productos colaterales.

De esta manera, el café constituye un patrimonio cultural que explica la dinámica económica, política, social y comunitaria de localidad con 
climas extremos, composición social migrante, economía solidaria y comercialización de productos y servicios tradicionales (Sales et ál., 2016). Es un símbolo de relación biosférica con el entorno, un eje central de la agenda local y un instrumento de ventajas competitivas para la planeación estratégica de su comercialización.

\section{Estudios del emprendimiento social}

En el marco del confinamiento de las personas debido a su condición de trabajadores y consumidores, el sector turístico y caficultor de la región huasteca se ha visto afectado hasta reducir en un $75 \%$ su actividad emprendedora y comercializadora. Los estudios que se han realizado en la localidad y la región destacan como ventaja competitiva de la comunidad a la identidad biosférica en torno al cuidado de sus atractivos y emblemas coloniales.

Sin embargo, los trabajos sobre el emprendimiento social se han edificado desde la optimización de recursos (oportunidades derivadas de políticas sociales de fomento empresarial o reactivación económica) hasta la innovación de procesos (generación de oportunidades desde las alianzas estratégicas entre los micro-, pequeños y medianos proyectos empresariales hasta las multinacionales que buscan insertar en el mercado local a cambio de que las mipymes accedan al mercado internacional).

Algunos trabajos destacan los proyectos colaborativos pensados desde visiones del mundo ambientalistas o ecologistas en donde el turismo cooperativo y la caficultura solidaria son emblemas distintivos de las regiones y ventajas competitivas en las decisiones de los visitantes (Bouls, 2020). Se trata de un turismo posmaterialista que busca relaciones con el entorno a partir de la colaboración y la fraternidad con sectores excluidos, marginados o vulnerados en sus derechos económicos, políticos o sociales.

Los trabajos relativos a las ecociudades, ecoaldeas y ecobarrios en la comercialización de productos orgánicos y huertas colectivas son proyectos 
emancipadores de la naturaleza que buscan el respeto a los derechos de las especies animales y vegetales por su coexistencia con los humanos. Son organizaciones de flujos migratorios y comunidades oriundas aliados por un mismo objetivo: preservar los recursos para las futuras generaciones.

En ese marco de recursos y futuro comunes, los proyectos agroindustriales se aproximan a su dimensión orgánica como sello de calidad para consumidores cada vez más conscientes de su poder adquisitivo y exigentes en cuanto a la calidad de los productos libres de transgénicos (Pérez, 2020). En esta naciente cultura vegana, los proyectos de emprendimiento contemplan su plena satisfacción a partir de las demostraciones de cultivo milenario y procesamiento libre de agroquímicos.

Otras investigaciones muestran como los proyectos ecoturistas tienden a ser cada vez más espirituales, orientados a las experiencias satisfactorias, la movilidad de cero emisiones y la felicidad eudaimónica (búsqueda de sentido de pertenencia con el entorno). Se sabe que aquellos turistas con un significado eudaimónico de la naturaleza no solo son leales a los destinos, sino que, además, generan redes solidarias de hospedaje, visitas guiadas y aprovisionamiento de recursos para preservar los recursos locales.

A diferencia del turismo hedonista (búsqueda de placer o displacer) el turismo eudaimónico se desenvuelve en zonas poco exploradas, comunitarias y frutales donde el placer de la estancia da origen al sentido de pertenencia y apego al lugar (Quiroz, 2020). El incremento de rituales y ceremoniales entre los turistas espirituales muestra una evidente tendencia hacia defensa de la naturaleza a partir del conocimiento de sus símbolos más que del consumo de sus recursos.

En resumen, los estudios del emprendimiento social en materia de agroindustria y turismo muestran un evidente cambio social indicado por el significado de la experiencia de ruptura con lo urbano, el traslado, el destino, la estancia y el retorno sistemático al lugar de pertenencia. En ese escenario, los proyectos de desarrollo local se han concentrado en la cooperación solidaria, el apoyo comunitario y la fraternidad con el entorno. 
Se trata de un contexto en el que turistas, migrantes y oriundos conviven en una atmosfera de significados que orienta sus decisiones de compra y venta.

\section{Especificación de un modelo para el estudio del emprendimiento social}

A partir de los marcos teóricos, conceptuales y empíricos revisados, es posible delinear los ejes y trayectorias de las relaciones entre las variables que explican el emprendimiento social como resultado de usos y costumbres cooperativistas, solidarios y fraternales entre comunidades, migrantes, turistas y entorno.

En el caso de la región Huasteca, los pueblos mágicos de Xilitla, Real de Catorce y Valles, el emprendimiento social puede ser explicado desde un eje central que va del biosferismo al posmaterialismo. Es decir, el sentido de pertenencia y apego al lugar parece complementarse con el sentido posmaterialista de turistas nacionales y extranjeros que sistemáticamente visitan y exploran los lugares emblemáticos y redescubren los centros ceremoniales. La convergencia de estas dos dimensiones hace suponer un continuum eudaimónico que radica en la búsqueda de espacios representados como recursos y futuros comunes.

Otro eje diferente es el que va del hedonismo o búsqueda del placer y displacer hacia el materialismo turista que se puede observar en el hacinamiento de parques acuáticos, cadenas de hoteles o restaurantes. Se trata de un sendero de costos y beneficios en los que la oferta y la demanda establece el volumen de visitas, consumo y divisas para la región, pero no garantiza en modo alguno el retorno de los mismos visitantes. Se considera que una mayor inversión y financiamiento a la infraestructura hotelera o recreativa permitirá incrementar sustancialmente el número de visitantes, pero al final son las promociones las que definen la crisis o prosperidad del sector.

Ambos ejes pueden ser comparados con el propósito de establecer las ventajas competitivas de la región en materia de turismo y caficultora, 
aunque la literatura consultada parece advertir que son complementarios. En virtud de que la promoción de las comunidades mágicas y caficultoras es una estrategia nacional, se espera que el hedonismo active las necesidades, gustos y preferencias, pero es el eudaimonismo el que define las expectativas de los visitantes.

Por consiguiente, están orientados hacia sectores diferentes, pero complementarios en cuanto a su poder de consumo e impacto para las zonas receptoras. Por lo tanto, las dimensiones teóricas de la percepción de emprendimiento se ajustan a las dimensiones ponderadas, las dimensiones empíricas ponderadas y son diferentes con respecto a estas.

\section{Método}

Diseño. Se llevó a cabo un estudio no experimental, trasversal y exploratorio.

Muestra. Se realizó una selección no probabilística de 300 microemprendedores, considerando su actividad principal orientada a la comercialización del café y derivados tales como dulces, bebidas y pan, así como su acceso a microfinanciamiento registrado en el padrón del municipio.

Instrumento. Se utilizó la escala de percepción de emprendimiento de García et ál., (2016) la cual incluye 20 ítems relativos a la percepción de oportunidad económica (p. ej. la promoción de los pueblos mágicos atraerá la inversión a Xilitla); percepción de oportunidad social (p. ej. recibiré el apoyo de mi familia al vender dulces de café); percepción de oportunidad ambiental (p. ej. el café se venderá en invierno por el frio de la localidad); percepción de oportunidad de financiamiento (p. ej. la venta del café será financiada por el gobierno local) y la percepción de oportunidad de ventas (p. ej. el café es una necesidad prioritaria para turistas). Cada ítem se contesta con alguna de cinco opciones que van desde 0 , para nada probable, hasta 5 , para muy probable. 
Procedimiento. Se utilizó la técnica Delphi para la homogenización de los significados de las palabras incluidas en los reactivos. Se garantizó el anonimato y confidencialidad por escrito en donde también se les informó acerca de que los resultados del estudio no afectarían su estatus económico, político, social o comunitario. Las encuestas se aplicaron en los establecimientos de comercialización del café y productos derivados. La información se procesó en el Paquete Estadístico para Ciencias Sociales (spss por sus siglas en inglés) y análisis de momentos estructurales (A Mos por sus siglas en ingles). Se estimaron la media, desviación estándar, alfa de Cronbach, chi cuadrada, кмо, pesos factoriales, betas, bondad de ajuste y residual.

\section{Resultados}

La consistencia interna de la escala general (alfa de 0.724) y las subescalas específicas (economía con alfa de 0.718 ; financiamiento con alfa de 0.796 ; ventas con alfa de 0.771 ; social con alfa de 0.701 ; ambiental con alfa de 0.703) alcanzaron valores alfa de Cronbach superiores a 0.700 (tabla 1). 
256 REVISTA ACTIVOS | Blanca Estela Bernal Escoto, Karen Michelle Rosete Herrera, Mayra Hayde Estrada Cabada

\begin{tabular}{|c|c|c|c|c|c|c|c|c|c|}
\hline 里 & & & & & & & & & \\
\hline 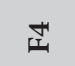 & & & & & & & & & \\
\hline$\cong$ & & & & & & & & & \\
\hline II & & & & & & & 察 & $\begin{array}{l}\text { जै } \\
\text { กै? }\end{array}$ & $\begin{array}{l}n \\
\tilde{n} \\
0\end{array}$ \\
\hline 正 & & 菑 & $\begin{array}{l}\text { ते } \\
\text { กิ }\end{array}$ & $\begin{array}{l}\vec{\sigma} \\
0 \\
0\end{array}$ & $\begin{array}{l}\text { 루 } \\
\text { ڤn } \\
0\end{array}$ & & & & \\
\hline$\frac{\pi}{\gtrless}$ & 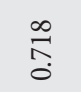 & 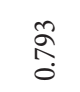 & 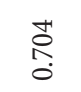 & $\underset{\widehat{N}}{\mathbb{N}}$ & $\stackrel{ }{\hat{N}}$ & 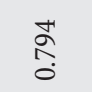 & $\begin{array}{l}\stackrel{+}{0} \\
\stackrel{2}{0}\end{array}$ & $\begin{array}{l}\stackrel{H}{\stackrel{2}{0}} \\
0\end{array}$ & $\begin{array}{l}\stackrel{\infty}{\infty} \\
\stackrel{0}{0}\end{array}$ \\
\hline 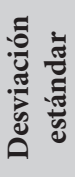 & & $\stackrel{\text { ô }}{\text {. }}$ & 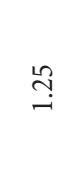 & 弇 & 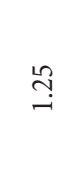 & & 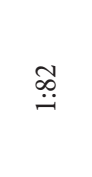 & 芦 & 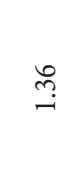 \\
\hline$\frac{\pi}{\overbrace{\pi}^{\pi}}$ & & $\vec{\sim}$ & $\underset{+}{\stackrel{H}{+}}$ & $\stackrel{\leftrightarrow}{\sigma}$ & $\vec{m}$ & & $\stackrel{\text { m }}{+}$ & $\underset{+}{\stackrel{\sim}{+}}$ & $\begin{array}{l}\vec{\infty} \\
+ \\
+\end{array}$ \\
\hline 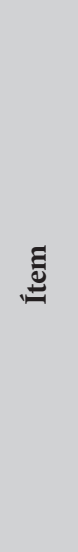 & 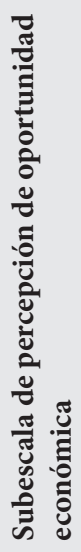 & 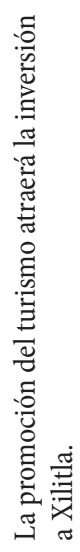 & 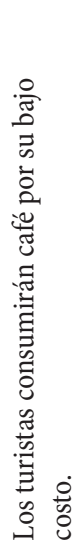 & 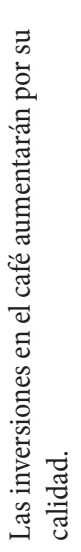 & 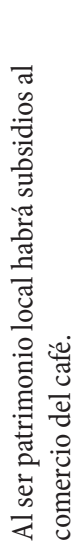 & 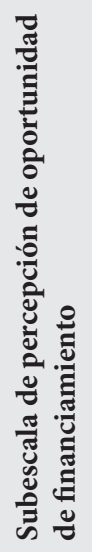 & 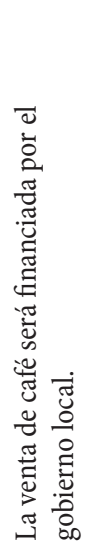 & 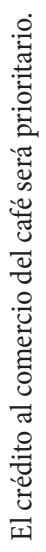 & 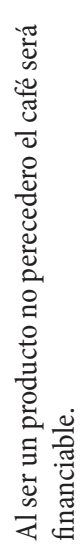 \\
\hline
\end{tabular}


Caracterización de las mipymes sostenibles del sector turístico | REVISTA ACTIVOS 257 del estado de Baja California, México

\begin{tabular}{|c|c|c|c|c|c|c|c|c|c|}
\hline in & & & & & & & & & \\
\hline 壱 & & & & & & & & 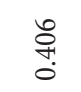 & 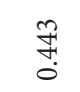 \\
\hline$\cong$ & & & $\begin{array}{l}\overrightarrow{\widetilde{N}} \\
\tilde{n}\end{array}$ & $\begin{array}{l}\infty \\
\substack{n \\
0}\end{array}$ & 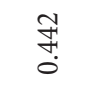 & $\begin{array}{l}\text { Fै } \\
0 \\
0\end{array}$ & & & \\
\hline$\widetilde{I}$ & $\begin{array}{l}\stackrel{n}{o} \\
\stackrel{0}{0}\end{array}$ & & & & & & & & \\
\hline $\overrightarrow{\text { 工 }}$ & & & & & & & & & \\
\hline$\stackrel{\frac{\pi}{Z}}{\frac{\pi}{4}}$ & $\begin{array}{l}\overline{\widehat{\Omega}} \\
\hat{\sigma}\end{array}$ & $\begin{array}{l}\hat{N} \\
\hat{0}\end{array}$ & $\stackrel{\widetilde{N}}{\stackrel{0}{0}}$ & 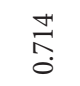 & 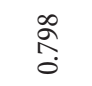 & $\stackrel{n}{\overparen{0}}$ & $\begin{array}{l}\vec{\circ} \\
\hat{0}\end{array}$ & 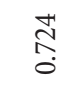 & $\begin{array}{l}\text { F } \\
\stackrel{5}{\circ}\end{array}$ \\
\hline 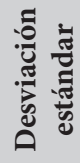 & $\stackrel{\text { Iִ }}{\text { I }}$ & & $\stackrel{\infty}{\stackrel{\infty}{n}}$ & $\stackrel{\simeq}{ت}$ & $\widetilde{\sim}$ & 논 & & 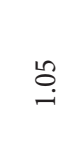 & f \\
\hline${ }^{\stackrel{\pi}{3}}$ & $\begin{array}{l}\stackrel{\leftrightarrow}{+} \\
\dot{+}\end{array}$ & & $\stackrel{\text { mे }}{+}$ & $\underset{+}{\stackrel{P}{+}}$ & $\stackrel{\infty}{\underset{+}{+}}$ & $\stackrel{\overbrace{}}{+}$ & & రָ & $\stackrel{\overbrace{}}{\overbrace{}}$ \\
\hline 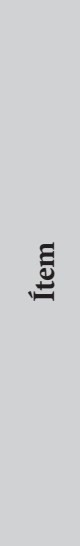 & 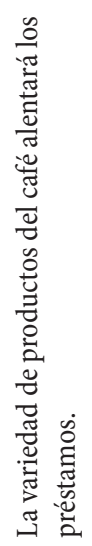 & 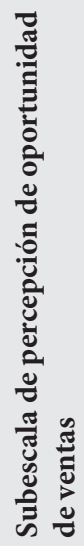 & 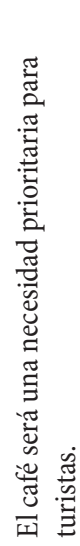 & 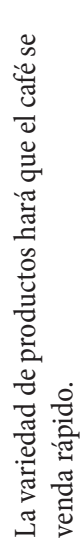 & 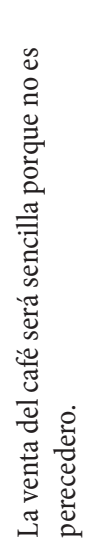 & 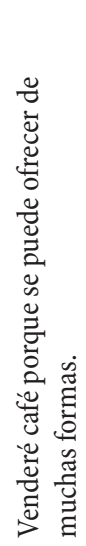 & 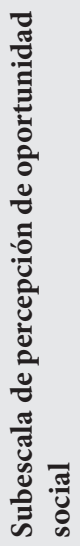 & 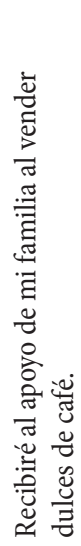 & 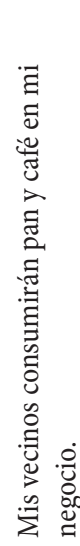 \\
\hline
\end{tabular}


258 REVISTA ACTIVOS | Blanca Estela Bernal Escoto, Karen Michelle Rosete Herrera, Mayra Hayde Estrada Cabada

\begin{tabular}{|c|c|c|c|c|c|c|c|c|}
\hline L 10 & & & & 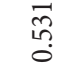 & 管 & 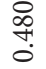 & $\vec{\Im}$ & تี \\
\hline 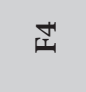 & की & $\begin{array}{l}\stackrel{0}{+} \\
\stackrel{+}{\circ}\end{array}$ & & & & & & 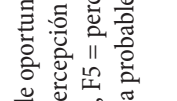 \\
\hline$\dddot{I}$ & & & & & & & & 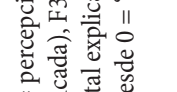 \\
\hline $\mathbb{I}$ & & & & & & & & 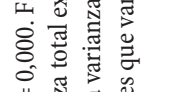 \\
\hline$\vec{I}$ & & & & & & & & 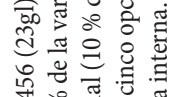 \\
\hline$\stackrel{\pi}{\sharp}$ & \begin{tabular}{l}
\multirow{2}{\infty}{} \\
$\stackrel{0}{0}$
\end{tabular} & $\begin{array}{l}\text { To } \\
\stackrel{0}{0}\end{array}$ & 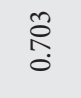 & $\begin{array}{l}\infty \\
\stackrel{\infty}{0} \\
0\end{array}$ & $\stackrel{\pi}{\stackrel{n}{0}}$ & 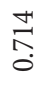 & 市 & 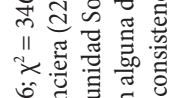 \\
\hline 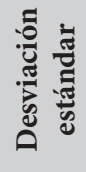 & $\stackrel{n}{m}$ & $\underset{ت}{\stackrel{*}{*}}$ & & $\stackrel{\text {. }}{.}$ & గి & $\stackrel{\text { 농 }}{2}$ & • & 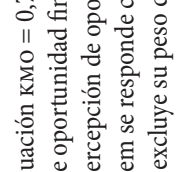 \\
\hline$\frac{\pi}{\tilde{3}}$ & $\stackrel{\infty}{\stackrel{\infty}{\rightarrow}}$ & 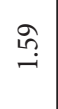 & & $\stackrel{+}{\text { S }}$ & $\widetilde{\Omega}$ & 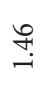 & $\underset{ָ}{\widetilde{Z}}$ & 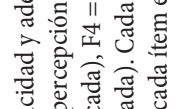 \\
\hline 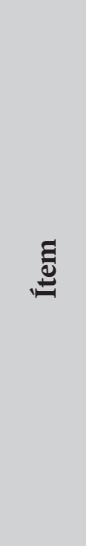 & 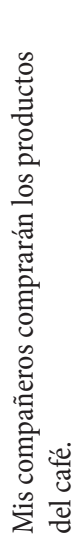 & 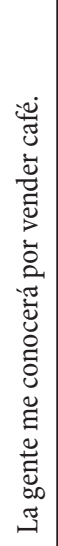 & 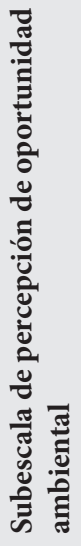 & 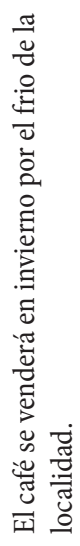 & 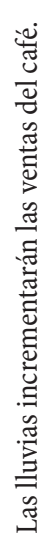 & 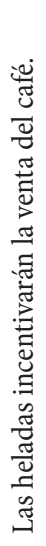 & 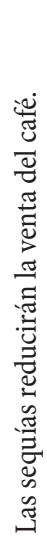 & 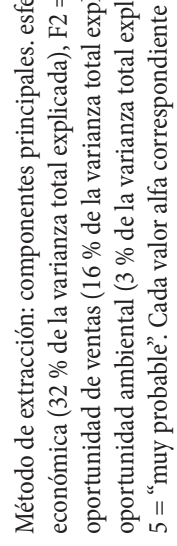 \\
\hline
\end{tabular}

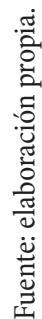


Respecto a la adecuación y la esfericidad кмо $=0,796 ; \chi^{2}=346,456$ (23gl) $\mathrm{p}=0.000$ estos alcanzaron valores mínimos indispensables para realizar el análisis factorial exploratorio de componentes principales con rotación varimax y establecer los factores correlacionados con ítems a partir de valores superiores a 0.300 .

Tabla 2. Relaciones de dependencia entre los factores y el constructo

\begin{tabular}{|l|c|l|c|c|c|c|}
\cline { 3 - 7 } \multicolumn{2}{c|}{} & Estimación & SE & CR & P \\
\hline Economía & $\leftarrow$ & Emprendimiento & .100 & & & \\
\hline Financiamiento & $\leftarrow$ & Emprendimiento & -.052 & .051 & -1.017 & .309 \\
\hline Ventas & $\leftarrow$ & Emprendimiento & -.091 & .071 & -1.289 & .197 \\
\hline Social & $\leftarrow$ & Emprendimiento & .156 & .122 & 1.277 & .202 \\
\hline Ambiental & $\leftarrow$ & Emprendimiento & .053 & .054 & .979 & .328 \\
\hline
\end{tabular}

Fuente: elaboración propia.

Se establecieron cinco factores relativos a economía (32\% de la varianza total explicada), financiamiento ( $22 \%$ de la varianza total explicada), venta ( $16 \%$ de la varianza total explicada), social ( $10 \%$ de la varianza total explicada) y ambiental (3\% de la varianza total explicada).

De acuerdo con la tabla 2 y la figura 1, por consiguiente, las relaciones de dependencia entre los factores establecidos y el constructo emergente indican que es el factor de percepción de oportunidad social el que refleja al constructo de percepción de emprendimiento $(\beta=0,47)$, seguido de la percepción de oportunidad económica $(\beta=0,30)$ y la percepción de oportunidad ambiental $(\beta=0,16)$.

Por último, los valores de bondad de ajuste y residual confirman la aceptación de la hipótesis nula en torno al ajuste de las dimensiones teóricas con respecto a las dimensiones empíricas de la percepción de emprendimiento $\chi^{2}=1,335(5 \mathrm{gl}) \mathrm{p}=0,935 ; \mathrm{GFI}=0,982 ;$ AGFI $=0,947 ; \mathrm{RMSEA}=0,000$. 
Figura 1. Modelo de relaciones de dependencia reflejantes

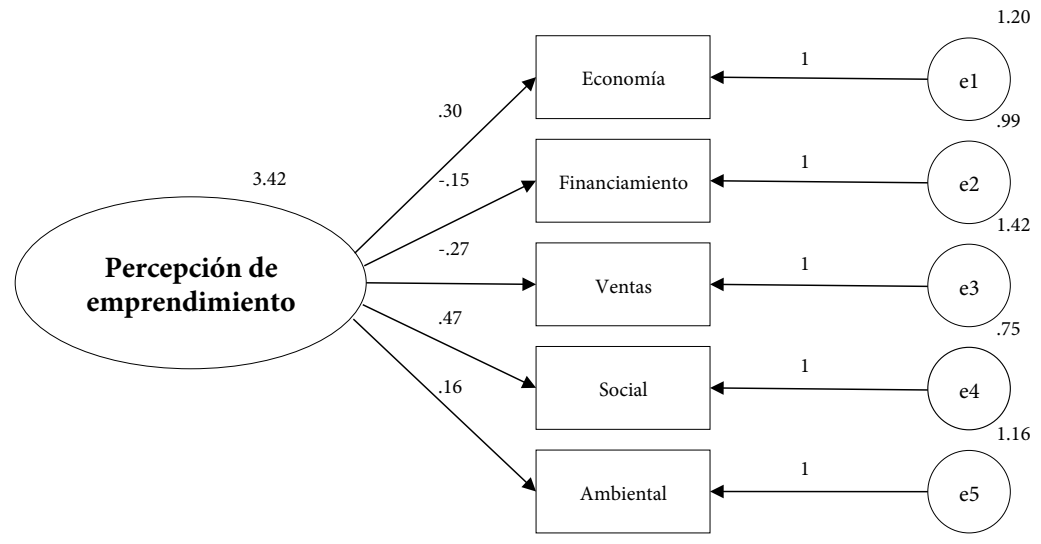

Fuente: elaboración propia.

\section{Discusión}

El objetivo del presente trabajo fue especificar un modelo para el estudio del emprendimiento caficultor en una región del centro de México distintivo por su denominación de pueblo mágico y ruta turística de la Huasteca Potosina.

Sin embargo, los hallazgos relativos a una estructura de cinco factores solo pueden ser considerados para la muestra de estudio, pues sugieren la extensión de la propuesta a otros escenarios de riesgo debido a la asimetría entre los actores políticos y privados, así como a la descoordinación entre los sectores públicos y privados en cuanto financiamiento se refiere. Líneas relativas a los efectos del crédito para el fomento empresarial y la gestión de cooperativas permitirá advertir diferencias y similitudes entre las estructuras de emprendimiento local y regional.

En virtud de que el emprendimiento social refleja la actividad caficultora, es menester orientar el financiamiento hacia la creación de redes cooperativas, empresas familiares o comunitarias con el fin de poder observar las 
diferencias en oportunidades y ventas en cada sector social. El desarrollo de investigaciones relativas a las asimetrías entre las organizaciones caficulturas por sus usos y costumbres, así como por sus tradiciones, permitirá anticipar escenarios de conflictos entre las partes interesadas.

En ese tenor, la teoría del emprendimiento social advierte que el turismo y la caficultura son oportunidades idóneas para el desarrollo local siempre que atiendan a sectores con poder adquisitivo, independientemente de su condición hedonista materialista o eudaimónica posmaterial.

El presente trabajo demostró que ambos ejes son convergentes en los proyectos de emprendimiento social cuando menos en sus dimensiones percibidas. Es decir, las expectativas de los flujos migratorios y comunidades oriundas parecen reflejar cinco dimensiones relativas a economía, financiamiento, ventas, solidaridad y ambientalismo, pero es la dimensión social la que refleja con mayor valor las expectativas caficulturas asociadas al turismo. Líneas de investigación concernientes a esta dimensión social; solidaridad, fraternidad, apoyo, compromiso, empatía, confianza, satisfacción y felicidad, permitirán extender el modelo hacia sectores que se distinguen por su compra orientada por su sentido de pertenencia al lugar.

Los estudios del emprendimiento social al poner énfasis en el hedonismo materialista y el eudaimonismo posmaterial han demostrado la complejidad del fenómeno al advertir relaciones de dependencia entre las dos dimensiones. Se trata de un proceso emergente a zonas y eventos de riesgo que parecen incentivar la ruptura con el habitad urbano, el placer del desplazamiento, la satisfacción con el servicio de hospedaje y el retorno sistemático a lugares no solo por su bajo costo sino por su significado compartido.

La especificación teórica, conceptual y empírica del modelo donde se destacan dos ejes uno del hedonismo al materialismo y otro del eudaimonismo al posmaterialismo parece enaltecer su convergencia en proyectos emprendedores de servicios turísticos y caficultores, con lo que se destaca este producto como el principal símbolo asociado a rupturas, travesías, 
estancias y retornos sistemáticos a lugares emblemáticos, inexplorados o ceremoniales. En el presente trabajo se ha destacado la convergencia de ambos ejes en un modelo de emprendimiento social que contempla la inclusión de las categorías. Futuros estudios relativos al análisis de comunidades y sectores implicados en ambos ejes permitirán anticipar escenarios de promoción, consumo y venta.

En síntesis, la exploración del emprendimiento caficultor en su dimensión social parece mostrar que coexisten dos ejes; uno que va del hedonismo al materialismo y otro que transita del eudaimonismo al posmaterialismo, los cuales deberán ser investigados como modelos de fomento empresarial o reactivación económica desde una planeación estratégica que desarrolle estas ventajas competitivas para beneficio local.

\section{Conclusión}

El aporte del presente trabajo al estado del conocimiento radica en el establecimiento de la confiabilidad y la validez de un instrumento que mide cinco factores relativos a la percepción de emprendimiento en una localidad del centro de México.

Futuras líneas de investigación con respecto a otros factores que el modelo no incluyó y tampoco estimó podrían llevarse a cabo si se considera que la comercialización de productos y servicios derivados del café es una actividad económica impulsada por políticas de fomento empresarial y microfinanciamiento, así como parte de los usos y costumbres sociales y comunitarios de la localidad de estudio. Además, las condiciones ambientales también juegan un papel decisivo en las percepciones de emprendimiento en general de productos y servicios agrícolas y las percepciones de oportunidad en torno al café.

En este sentido, la validez ecológica que consiste en la observación sistemática de los usos y costumbres, así como su correlación con escalas que miden las percepciones de oportunidad, brindarían una perspectiva 
integral del fenómeno que supone el café, su entorno, administración, comercialización y consumo.

Por consiguiente, la especificación de un modelo y la estimación de este en torno a indicadores que expliquen las particularidades de las localidades emprendedoras circunscritas al café es menester, ya que, si bien el emprendimiento supone una estructura de percepciones, decisiones y estrategias generales, el éxito de los proyectos depende de la especificidad local. Este es el caso de los sectores dedicados a la venta del café y la comercialización de sus productos y servicios derivados.

Ahora bien, las características de cada sector obligan a un análisis minucioso acerca de los usos y costumbres que hacen posible la subsistencia mediante la venta del café. En este sentido, las jefas de familia son un ejemplo de los grupos dedicados a la venta al menudeo y el emprendimiento diversificado de productos que incluyen al café.

Finalmente, la exploración de valores, percepciones y creencias de las jefas de familia dedicadas al café permitirá explicar y anticipar escenarios de crisis ambiental, económica, política y social, pero también de resiliencia y emprendimiento social.

\section{Referencias}

Acar, Z. y Acar, P. (2014). Organizational culture types and their effects on organizational performance in Turkish hospitals. Emerging Markets Journal, 3(3), 1-15. Dor: https://doi.org/10.5195/emaj.2014.47

Adams, S. (2020). Academic framework of social entrepreneurship. International Journal of Research Aspects of Engineering \& Management, 16(2), 1-6.

Anguiano, F. (2020). Meta-analysis of the effects of entrepreneurship on local development: Implications for the covid-19 coronavirus pandemic. International Journal of Research in Engineering 6 Science, 8(9), 40-47.

Anicijevic, N. (2013). The mutual impact of organizational culture and structure. Economic Annals, 58(198), 35-60. 
Bouls, B. (2020). Specification of a model of entrepreneurship: from opportunism to innovation. International Journal of Advanced Research, 8(3), 20-24.

Bustos, J. M. (2020). Una revisión de hallazgos en torno al desarrollo local. Eureka, 17(1), 141-161.

Carreón, J., Hernández, J., García, C., García, E., Rosas, F. y Aguilar, J. (2014). Especificación de un modelo de emprendimiento digital para el desarrollo humano mediante el uso intensivo de tecnologías de información y comunicación. Perspectivas Rurales, 13(25), 123-155.

Coronado, O. (2019). Gobernanza del emprendimiento social responsable. Nomadas, 57(1), 1-23.

Cruz, O., Arroyo, P. y Marmolejo, J. (2016). Innovaciones tecnológicas en la logística: gestión de inventarios, sistemas de información y terciarización de operaciones. En M, Quintero., Sales, J. y Velázquez, E. (coords.). Innovación y tecnología retos para su aplicación práctica en las empresas (pp. 165-178). México: Uaemex.

Escobar, R. (2014). Redes neuronales, procesos cognoscitivos y análisis de la conducta. Revista Internacional de Conductismo, 2(1), 23-43.

García, C. (2014). Especificación de un modelo de emprendedurismo social. Razón y Palabra, 18(88), 1-40.

García, C. (2015). Especificación de un modelo de emprendimiento electrónico. Ciencias en la Frontera, 13, 27-41.

García, C. (2020). Specification a model for study of entrepreneurship. Advanced Research Journal of multidisciplinary Discoveries, 49(1), 1-4.

García, C., Carreón, J., Hernández, J. y Salinas, R. (2016). Gobernanza de los actores y redes de innovación tecnológica. En M, Quintero., Sales, J. y Velázquez, E. (coords.). Innovación y tecnología retos para su aplicación práctica en las empresas. (pp. 79-94). México: Uaemex.

García, C., Carreón, J., Rosas, F., Aguilar, J. y Rivera, B. (2014). Especificación de un modelo de emprendimiento ciberpolítico. Perspectivas, 9, 30-67.

García, C., Valdés, O., Sánchez, R., Elizarraráz, G., Méndez, A. y Hernández, J. (2015). Diferencias entre emprendedores internautas con respecto a empatía, percepciones de riesgo y uso de aplicaciones tecnológicas. Revista Prospectivas de Psicología, 2(1), 68-85. 
Hernández, A. y Valencia, R. (2016). Instrumentos de innovación: las redes sociales en la internalización de las micro, pequeñas y medianas empresas mexicanas. En M, Quintero., Sales, J. y Velázquez, E. (coords.). Innovación y tecnología retos para su aplicación práctica en las empresas (pp. 47-66). México: Uaemex.

Instituto Nacional de Estadística, Geografía e Informática. (2010). XI Censo Nacional de Población. Aguascalientes: INEGI

Juárez, M. (2020). Specification a model for study of corporate assistance. Global Journal of Archeology 6 Anthropology, 11(2), 50-53.

Mendoza, E., Ramírez, L. y Atriano, R. (2016). Uso de los medios y las tecnologías en la creación de un sistema de innovación para el bien común. En $\mathrm{M}$, Quintero, Sales, J. y Velázquez, E. (coords.). Innovación y tecnología retos para su aplicación práctica en las empresas (pp. 95-114). México: Uaemex.

Molina, H. D. (2020). Representaciones socioambientales periurbanas. Kulxulkav, 26(54), 5-12.

Omotayo, O. y Adenike, A. (2013). Impact of organizational culture on human resource practices: a study of selected Nigerian private universities. Journal ofCompetitiveness, 5(4), 115-133. Dor: https://doi.org/10.7441/joc.2013.04.07

Pérez, G. (2020). Dimensional meta-analysis of trust: implications for covid-19 communication. Technium Social Science Journal, 5(1), 150-156.

Quintero, M., Velázquez, E., Sales, J. y Padilla, S. (2016). Una revisión del estado del arte sobre pymes. ¿y los estudios de innovación? En M, Quintero., Sales, J. y Velázquez, E. (coords.). Innovación y tecnología retos para su aplicación práctica en las empresas (pp. 31-43). México: Uaemex.

Quiroz, C. Y. (2020). Specification of a model of digital entrepreneurship. Current Research in Psychology \& Behavioral Sciences, 1(1), 1-4.

Robles, C., Alviter, L., Ortega, A. y Martínez, E. (2016). Cultura de calidad e innovación en la microempresa. En M, Quintero., Sales, J. y Velázquez, E. (coord.). Innovación y tecnología retos para su aplicación práctica en las empresas (pp. 11-30). México: Uaemex.

Saansongu, E. y Ngutor, D. (2012). The influence of corporate culture of employee commitment to the organization. International Journal of Business and Management, 7(22), 1-8.

Sales, J., Quintero, M. y Velázquez, E. (2016). Adaptación versus innovación: la formación de distritos industriales a partir de comunidades campesinas. 
266 REVISTA ACTIVOS | Blanca Estela Bernal Escoto, Karen Michelle Rosete Herrera, Mayra Hayde Estrada Cabada

Santa Cruz Atizapan y Chiconcuac. En M, Quintero., Sales, J. y Velázquez, E. (coords.). Innovación y tecnología retos para su aplicación práctica en las empresas (pp. 181-199). México: Uaemex.

Sandoval, F. R. (2020). Job expectations in the face of risks events and collateral social effects. International Journal of Humanities \& Social Science Educations, 7(5), 1-7.

Vázquez, C., Barrientos, B., Quintero, M. y Velázquez, E. (2016). Apoyos gubernamentales para la innovación, tecnología y capacitación de las pequeñas y medianas empresas en México. En M, Quintero., Sales, J. y Velázquez, E. (coord.). Innovación y tecnología retos para su aplicación práctica en las empresas (pp. 67-78). México: Uaemex. 